\title{
Surface Microstructure Study of Polyethylene Blends for Developing Environmental Degradable Plastic Bags
}

\author{
Ruhul Amin M. ${ }^{1 *}$, Basel F. Abu-Sharkh² and Mamdouh Al-Harthi ${ }^{2}$ \\ ${ }^{1}$ Department of Chemical Engineering \\ Bangladesh University of Engineering \& Technology (BUET), Dhaka-1000, Bangladesh \\ ${ }^{2}$ King Fahd University of Petroleum \& Minerals, \\ P. O. Box 5050 Dhahran 31261, Kingdom of Saudi Arabia
}

\begin{abstract}
The morphology of the polymer blends retrieved from degradation experiments was investigated by studying the fractured surfaces using Scanning Electron Microscope for investigating the degradability of environmentally degradable plastic bags. We study the influence of adding photodegradable additives to film grade polyethylene under natural weathering conditions in Saudi Arabia. The films were exposed in outdoor environments located in Dhahran, Saudi Arabia. Comparison of different additives was determined and the additive PDQ-H shows the highest performance on the basis of degradation rate in the harsh environment of Saudi Arabian desert.
\end{abstract}

Keywords: Degradation; polyethylene; additive; starch; polymer blends; SEM; DS

\section{Introduction}

Plastic objects have slowly substituted glass, paper and metals in several fields of application because of their low production cost, good physical properties and lightweight. But the current huge global production of plastics has generated enormous environmental concerns, mainly related to the waste generation by plastic packaging. Where recovery of plastics is not economically feasible, plastics often remain as litter. The market for biodegradable polymers is at this moment focusing on products in which biodegradability provides beneficial effects and customer acceptance. In order to be considered environmentally degradable, a plastic must disintegrate mechanically, which means that the molecular weight of the molecules must be reduced, and then these molecular fragments must be microbially susceptible.

Rodlan-Carrillo et al. studied starch metabolites and enzymes during starch-based plastic polymer biodegradation. ${ }^{1}$ hotodegradable polymers are synthetic polymers incorporating light-sensitive chemical additives or copolymers for the purposes of weakening the bonds of the polymer in the presence of ultraviolet radiation. Willow Ridge Inc., USA, produces a masterbatch that simultaneously triggers photodegradation and thermal-oxidative breakdown in PE. Willow Ridge products such as PolyStarch N, a masterbatch of 55\% cornstarch with LLDPE and is typically used at a 3\% level. ${ }^{2}$ Freedman et al. investigated the effect of adding $1.0 \%$ of $\mathrm{N}$-halogen compounds to polystyrene, polypropylene and polyethylene and found that it enhanced the photodegradability of the polymers in film form. ${ }^{3}$ Hill reported fully degradable plastic bags from polyethene that contain a degradable compostable plastic additive technology that induces reduction of the

* Corresponding Author: Ruhul Amin M

Email: $\underline{\text { amin@eche.buet.ac.bd }}$ plastic to carbon dioxide and water in just a few weeks. ${ }^{4}$ The environmental degradation of polyethylene was reviewed by Hakkarainen \& Albertsson. ${ }^{5}$ Various studies have shown that starch-based plastics do biodegrade under controlled composting conditions. One study by Chiellini et al. ${ }^{6}$ found that the resin did undergo ultimate biodegradation (i.e. mineralization) in simulated soil burial but not readily in composting conditions.

The thermal properties of a series of commercially degradable polymers were studied using thermogravimetry and differential scanning calorimetry by Day et al. ${ }^{7}$ Goheen et al. ${ }^{8}$ investigated low-density polyethylene films mixed with different percentages of corn starch which were exposed to soils and monitored for starch removal and chemical changes of the matrix using FTIR spectroscopy. It was reviewed the current trends in degradable plastics and technologies of two types of photodegradable plastics and the benzophenone-based additive ${ }^{9}$. Characteristic properties of modified polyethylene and polypropylene resins were shown. A mechanism of degradation was described briefly.

Hakozaki et al. reviewed the background and the present status of the technology of biodegradable and photodegradable plastics. The effect of the method of disposal on degradation was investigated. Special attention is paid to possible reuse of plastics and several examples are given ${ }^{10}$. Wiles et al. devised additive packages which, when added to polyolefins, can be used to fabricate films which are suitable for landfill covers, refuse sacks, etc., or, in a different manifestation, are suitable for compost bags ${ }^{11}$.

Billingham reviewed some studies and applications of oxo-biodegradable additives that can be incorporated 
into conventional hydrocarbon polymers inducing accelerated oxidation on exposure to light or heat ${ }^{12}$. Shah et al. ${ }^{13}$ characterized the initial degradation mechanism of starch filled LDPE which show that $\alpha$ amylase acts on the surface starch to cause cracks, holes, pitting and erosion which increase the surface area.

In this study, we investigate the degradation products and determine the time required to convert the material into gases or low molecular weight hydrocarbons. Plastic films were prepared by first premixing the film grade polyethylene pellets with different amounts of additives. The films were exposed in outdoor environments located in Dhahran, Saudi Arabia. Samples of the films were drawn at regular intervals and evaluated for degradation.

\section{Experimental}

\subsection{Materials}

Materials utilized in this investigation include film grade low density polyethylene and additives that enhance photo-degradation and biodegradation was supplied by Willow Ridge Co., USA, these are PDQ-H, Polystarch N and Polystarch Plus H.

\subsection{Sample Preparation}

Plastic films were prepared by blending different amounts of additives such as 10, 20, 30, 40 and 50 percent mixed with pure polyethylene. Subsequently, the mixer was fed to an extruder to produces the film.

\section{Extent of Miscibility of the Polymers with the Additives}

Differential Scanning Calorimetry (Model: DSCQ1000, Universal V4.2E TA Instruments) was used to quantify the extent of homogeneity of the polymers with the additives.

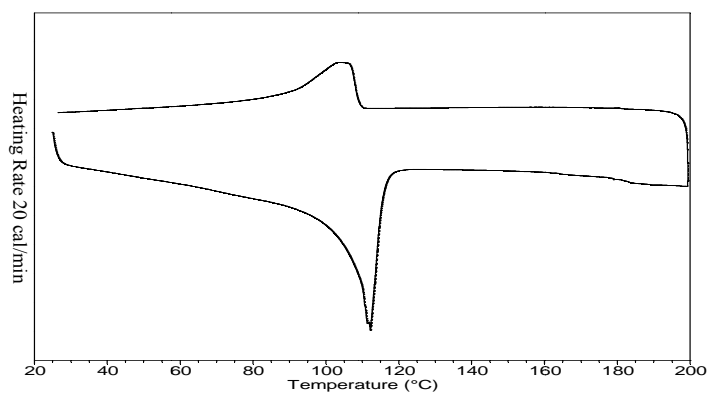

Fig.1. DSC analysis of sample mixed with $30 \%$ Polystarch-plus-H additive. The sample was heated at a rate of $20 \mathrm{cal}$ per min from $25^{\circ} \mathrm{C}$ to $200^{\circ} \mathrm{C}$. The upper curve is for heating and the lower curve is for cooling.
Fig.1 shows the heating and cooling curves obtained from DSC analysis. The heating and cooling curves are smooth and continuous which means that the additive is homogeneously mixed with polyethylene. After confirming the homogeneity of all the films, the samples were exposed in natural weathering condition for degradability studies.

\subsection{Degradation Studies}

The degradability of the films in outdoor environment was determined in this study. The films were exposed in outdoor environments at the exposure site located in Dhahran, Saudi Arabia. The films were exposed for one year and samples were drawn at regular intervals and evaluated for degradation.

\subsection{Sample Analysis}

The morphology of the blends retrieved from degradation experiments was investigated by studying the fractured surfaces using Scanning Electron Microscope (Model: JEOL JSM 5800LV). Samples were coated with a thin layer of carbon using a Carbon Evaporator in order to avoid sample charging during imaging. The imaging was performed at varying magnifications in (secondary electron mode) using an accelerating voltage of $10 \mathrm{kV}$. The chemical composition of samples was analyzed using an energy dispersive X-ray spectrometer fitted with an ultra-thin Be window.

\section{Results \& Discussion}

The SEM images obtained at different exposure time show that initially the degradation starts at a point and gradually the affected area increases with time ultimately covering the entire film. The SEM images for different exposure time $(0,30,120$ and 180 days) are presented in Fig. 2-7. Initially it is clear that there is no crack on the surface of the blend.

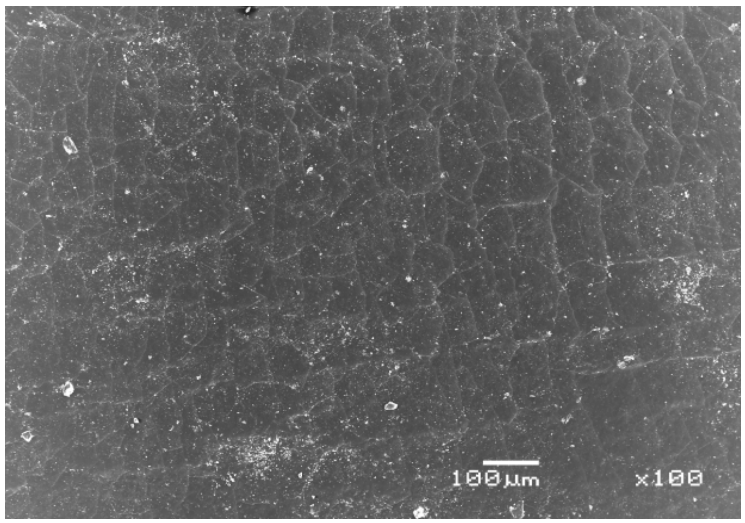

Fig.2. Fresh sample without additive 


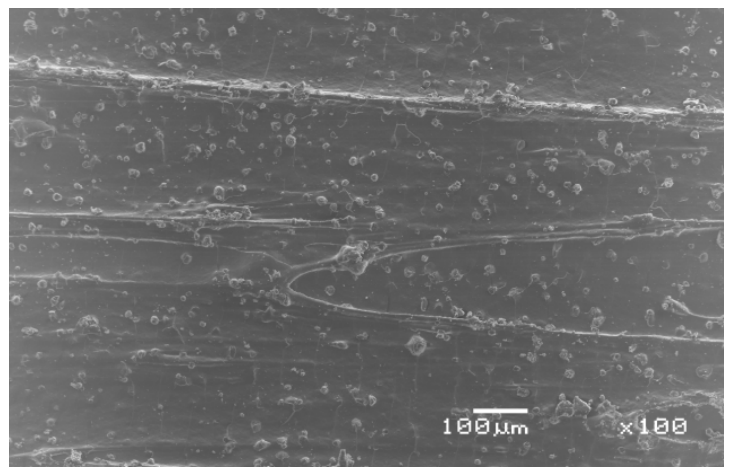

Fig. 3. SEM image of $10 \%$ Polystarch $\mathrm{H}$ after 30 days

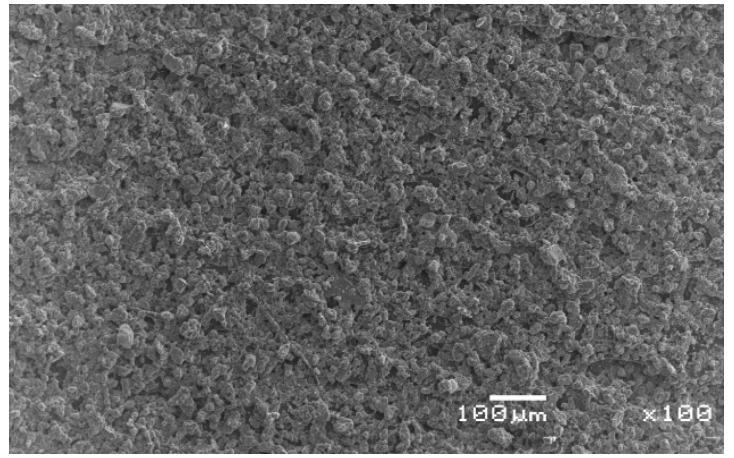

Fig.4. SEM image of 20\%Polystarch $\mathrm{H}$ after 120 days

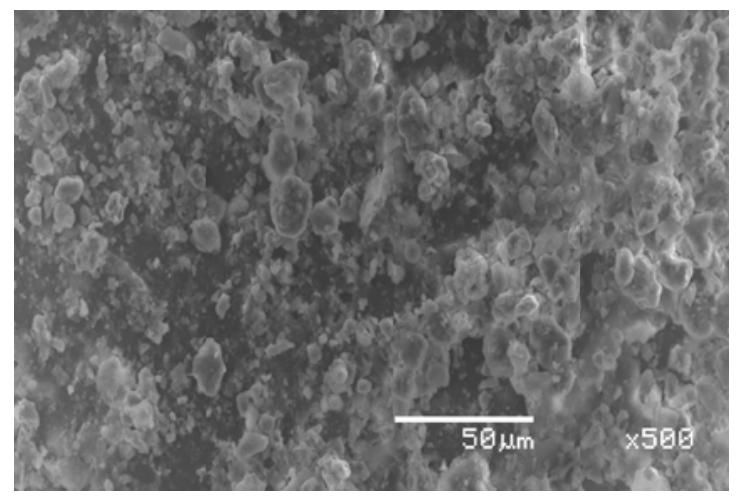

Fig.5. SEM image of $20 \%$ Polystarch N after 180 days

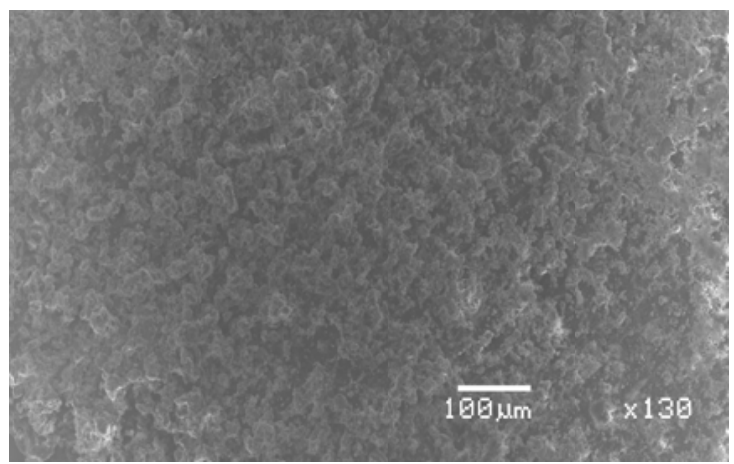

Fig.6. SEM image of $40 \%$ Polystarch $\mathrm{N}$ after 180 days

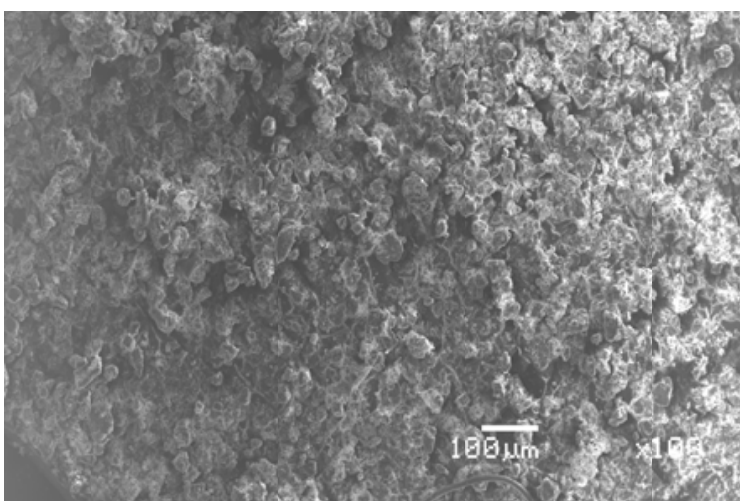

Fig.7. SEM image of 10\% PDQH after 180 days

It was observed that when the time of exposure increases, degradation of the material starts and gradually the affected area increases. For the material after 50 days of exposure, initiation of cracks and degradation occurs; after 95 days of exposure, degradation propagates rapidly, bigger cracks are shown as white area while some parts are still unaffected shown as black area and after 120 days of exposure, very big cracks and degradation took place for almost all parts of the blend. This degradation is due to the degradation of the starch as well as the oxidized the LDPE amorphous regions. Thereby, the surface area of polymer blends increases and this improves oxygenbased reactions that enhances and increases LDPE chain oxidation reaction.

From this it's clear that when compared to PN30 and $\mathrm{PH} 30$, the degradation is higher for the blends containing PDQ-H. This is due to the variation in the nature of the additives used for this study. Here the polystarch $\mathrm{N}$ contains only polyethylene and corn starch, while polystarch $\mathrm{H}$ consists of some UV and oxidative prodegradants which increases the degradation rate. However the PDQ-H is a non-starch based additive. This additive enhances the degradation via photo (UV) and oxidative methods thereby causing a drastic decrease in the molecular weight of the blends.

\section{Conclusion}

1. The additive PDQ-H shows the highest performance on the basis of degradation rate in the harsh environment of Saudi Arabian desert.

2. Samples become porous and start to be taken away by the flowing air after two months.

3. After 4 months, cracked, fractured big pits were found which indicate severe photo-degradation.

4. Therefore, from this study, it can be concluded that within 8 to 9 months, all the samples were almost completely degraded. 


\section{Acknowledgements}

This work was funded by King Abdul Aziz City for Science and Technology (KACST) (Grant Number: 7925).

\section{References}

1. Roldan, T.; Rodriguez, R.; Diaz, D.; Vazquez, H.; Manzur, A.; Torres, A. Bioresource Technology 2003, 86, 1 .

2. Leaversuch, B. Plastics Technology 2002, 88, 15.

3. Freedman, B. Journal of Applied Polymer Science 1976, 20, 911.

4. Hill, S. Materials World 1999, 7, 135.

5. Hakkarainen, M.; Albertsson, A. Advances in Polymer Science 2004,169, 177.

6. Chiellini, E.; Corti, A.; Swift, G. Polymer Degradation and Stability 2003, 81, 341.

7. Day, M.; Cooney, J. D.; Shaw, K.; Watts, J. Journal of Thermal Analysis and Calorimetry 1998, 52, 261.

8. Goheen, S. M.; Wool, R. P. J Appl Polym Sci 2003, 42, 2691.
9. Iwasa, Satoru. Harima Chem., Inc., Kakogawa, Photodegradable plastics (1990), JETI 38(10), 6870.

10. Hakozaki, Junichi; Ishikawa, Yoshihiro. (1990), Development in the technology of degradable plastics, JETI 38(3), 52-6.

11. Wiles, D. M.; Cermak, B. E.; Gho, J. G.; Hare, C. W. J. (1998), "Controlled - lifetime, environmentally degradable plastics based on conventional polyethylenes". Addcon World '98: Additives for the New Millennium, Official Book of Papers, International Plastics Additives and Modifiers Conference, London, Nov. 9-10, 1998 Paper 14/1-Paper 14/6. Publisher: Rapra Technology, Shrewsbury, UK.

12. Billingham, N. C.; Wiles, D. M.; Cermak, B. E.; Gho, J. G.; Hare, C. W. J.; Tung, J. F. (2000), "Controlled-lifetime environmentally degradable plastics based on conventional polymers" Addcon World 2000, Two-Day Conference, 6th, Basel, Switzerland, Oct. 25-26, 2000 paper16/ 1paper16/6. Publisher: Rapra Technology Ltd., Shrewsbury, UK.

13. Shah, P.B.; Bandopadhyay, S.; Bellare, J.R. Polymer Degradation and Stability 1995, 47, 165. 